Doug Geisler, Eva K. Grebel, and Dante Minniti, eds.

\title{
Towards Better Age Estimates for Stellar Populations: The $Y^{2}$ Isochrones
}

\author{
Pierre Demarque \\ Department of Astronomy, Yale University, PO Box 208101, New \\ Haven, CT 06520-8101, USA \\ demarque@astro.yale.edu
}

Sukyoung Yi

Center for Space Astrophysics, Yonsei University, Seoul 120-749, Korea and

California Institute of Technology, Mail Code 405-47, Pasadena, CA

91125, USA

yi@srl.caltech.edu

Yong-Cheol Kim

Center for Space Astrophysics, Yonsei University, Seoul 120-749, Korea kim@csa.yonsei.ac.kr

Abstract. New theoretical isochrones are presented and compared to the observational data.

\section{Isochrone Parameters}

We have constructed a new set of isochrones, called the $Y^{2}$ isochrones, that represent an update of the Revised Yale Isochrones (RYI), using improved opacities and equations of state (OPAL, Iglesias \& Rogers 1996; Rogers, Swenson, \& Iglesias 1996), energy generation rates (Bahcall \& Pinsonneault 1992) and neutrino loss rates (Itoh et al. 1989). Helium diffusion (Thoul, Bahcall \& Loeb 1994 ) and convective core overshoot have also been taken into account.

The heavy element abundance ranges from $Z=0.0001$ to 0.08 , with the corresponding helium content $\mathrm{Y}$ varying between 0.23002 and 0.35000 , corresponding to $\Delta Y / \Delta Z=2$. The ages range from $1 \mathrm{Myr}$ to $20 \mathrm{Gyr}$.

The first set of isochrones is for the scaled solar mixture (Yi et al. 2001). A subsequent paper considers the effects of $\alpha$-element enhancement, believed to be relevant in many stellar systems.

Two additionally significant features of these isochrones are that (1) the stellar models start their evolution from the pre- main sequence birthline instead of from the zero-age main sequence, and (2) the color transformation has been performed using both the latest table of Lejeune, Cuisinier \& Buser $(1997,1998)$, and the older, but now modified, Green, Demarque \& King (1987) table. 


\section{Results}

The poster presented comparisons with observed cluster data. The change in the derived ages of star clusters caused by this update in stellar models depend sensitively on their metallicity. Whereas globular cluster ages are decreased by amounts between $15 \%$ (for $\mathrm{Z}=0.0004$ ) and $11 \%$ (for $\mathrm{Z}=0.004$ ) relative to $\mathrm{RYI}$ based studies, the ages of solar metallicity $(\mathrm{Z}=0.02)$ star clusters are increased by about $10 \%$. When a simple scaling for $\alpha$-element enhancement similar to that used by Chaboyer et al. (1992) is taken into account as well, the reduction in the ages of the most metal poor globular clusters is approximately $20 \%$.

When post-RGB evolutionary stages are included, we find that the ages of globular clusters derived from integrated colors are consistent with the isochrone fitting ages.

\section{Summary}

A more detailed description of the $Y^{2}$ isochrones can be found in Yi et al. (2001). Isochrone tables and interpolation software can be obtained at the website http://achee.srl.caltech.edu/y2solarmixture.htm, or by contacting one of the authors by e-mail.

\section{Acknowledgements}

This research was supported in part by NASA grant NAG5-8406 (PD), and the Creative Research Initiative Program of the Korean Ministry of Science \& Technology (SY, YCK).

\section{References}

Bahcall, J.N. \& Pinsonneault, M.H. 1992, Rev.Mod.Phys. 60, 297; and private communications.

Chaboyer, B., Deliyannis, C.P., Demarque, P., Pinsonneault, M.H. \& Sarajedini, A. 1992, ApJ, 388, 372

Green, E.M., Demarque, P. \& King, C.R. 1987, the Revised Yale Isochrones and Luminosity Functions (New Haven: Yale Univ. Obs.)

Iglesias, C.A. \& Rogers, F.J. 1996, ApJ, 464, 943

Itoh, N., Adachi, T., Nagakawa, M., Kohyama, Y. \& Munakata, H. 1989, ApJ, 339, 354; erratum ApJ, 360, 741

Lejeune, Th., Cuisinier, F. \& Buser, R. 1997, A\&A, 125, 229

Lejeune, Th., Cuisinier, F. \& Buser, R. 1998, A\&A, 130, 65

Rogers, F.J., Swenson, F.J. \& Iglesias, C.A. 1996, ApJ, 456, 902

Thoul, A.A., Bahcall, J.N. \& Loeb, A. 1994, ApJ, 421, 828

Yi, S., Demarque, P., Kim, Y.-C., Lee, Y.-W., Ree, C.-H., Lejeune, Th. \& Barnes, S. 2001, ApJS, in press (Astro-ph/0104292) 